\title{
P03-06. Preserved cellular and humoral immune responses in macaques infected with SIVmac239 and subjected to early antiretroviral therapy B Makitalo ${ }^{3}$, L Walther-Jallow ${ }^{3}$, G Ozkaya Sahin ${ }^{1}$, J Michaelsson ${ }^{3}$, M Spangberg ${ }^{2}$, R Thorstensson ${ }^{2}$, E Fenyo ${ }^{1}$ and A Spetz*3
}

Address: ${ }^{1}$ Department of Laboratory Medicine, Lund University, Lund, Sweden, ${ }^{2}$ Swedish Institute for Infectious Disease Control, Stockholm, Sweden and ${ }^{3}$ CIM, Department of Medicine, Karolinska University Hospital Huddinge, Karolinska Institutet, Stockholm, Sweden

* Corresponding author

from AIDS Vaccine 2009

Paris, France. 19-22 October 2009

Published: 22 October 2009

Retrovirology 2009, 6(Suppl 3):P23 doi:10.1186/1742-4690-6-S3-P23

This abstract is available from: http://www.retrovirology.com/content/6/S3/P23

(c) 2009 Makitalo et al; licensee BioMed Central Ltd.

\section{Background}

The kinetics of cellular and humoral immune responses was evaluated in a macaque model after early introduction of antiretroviral treatment. Twelve cynomolgus macaques were infected intravenously with the patogenic SIVmac239 strain. Treatment with the antiretroviral drug Tenofovir was initiated 10 days after inoculation with SIVmac239 and was administered subcutaneously as daily injections for 4 months.

\section{Methods}

Viral load, CD4 counts and cellular immune responses were assessed by measuring proliferation and cytokine production by ELISpot as well as intracellular cytokine staining. Flow cytometry analyses were performed to assess $\mathrm{T}$ cell activation and memory $\mathrm{T}$ cell populations. In addition, the emergence of neutralizing antibodies was monitored throughout the study.

\section{Results}

Unexpectedly many macaques were able to keep good viral control (7/12) for 16 months after stopping Tenofovir and kept their CD4 T cell numbers. The majority of animals had substantial immune responses in terms of proliferative capacity and IFN-gamma production upon re-stimulation with SIV antigens in vitro. In addition, we demonstrate that infection of 12 cynomolgus macaques with SIVmac239 leads to early induction of neutralizing antibodies against SIVsm (strain SMM3). Neutralizing titres exceeded 1:640 in all monkeys by 4.5 months post infection and were as high as 1:10240 in three monkeys.

\section{Conclusion}

Early initiation (here 10 days) of effective antiretroviral treatment seem to have preserved cellular immune responses in terms of cell numbers, proliferative capacity and IFN-gamma production. It is conceivable that cellular immune responses may have contributed to the long-term control of viral load in a large proportion (7/12) of animals in the present study and in addition have provided sufficient help for induction of neutralizing antibodies. 